\title{
On the motion of linked spheres in a Stokes flow
}

\author{
F. Box ${ }^{1,2} \cdot$ E. Han ${ }^{1,3} \cdot$ C. R. Tipton ${ }^{1,4} \cdot$ T. Mullin ${ }^{1,2}$
}

Received: 8 December 2016 / Revised: 12 February 2017 / Accepted: 14 February 2017 / Published online: 18 March 2017

(C) The Author(s) 2017. This article is published with open access at Springerlink.com

\begin{abstract}
The results of an experimental investigation into the motion of linked spheres at low Reynolds number are presented. Small permanent magnets were embedded in the spheres and torques were generated by application of an external magnetic field. Pairs of neutrally buoyant spheres, connected by either glass rods or thin elastic struts, move in a reciprocal orbit when driven by an oscillatory field. An array of three spheres linked by elastic struts buckles in a periodic, non-reciprocal manner. The induced magneto-elastic buckling propels the elemental swimmer and we find that the geometrical asymmetry of the device, introduced by the struts of different lengths, determines the swimming direction. We propose that this novel method of creating movement remotely is suitable for miniaturization.
\end{abstract}

Keywords Swimming - Torsional oscillations - Stokes flow $\cdot$ Magnetic actuation

F. Box

box@maths.ox.ac.uk

1 Manchester Centre for Nonlinear Dynamics, School of Physics and Astronomy, University of Manchester, Oxford Road, Manchester M13 9PL, UK

2 Present Address: Mathematical Institute, University of Oxford, Andrew Wiles Building, Radcliffe Observatory Quarter, Woodstock Road, Oxford OX2 6GG, UK

3 Present Address: James Franck Institute, The University of Chicago, Chicago, IL 60637, USA

4 Present Address: Johnson Matthey Oil and Gas, Measurement Technology Centre, The Moat, Belasis Hall Technology Park, Billingham TS23 4ED, UK

\section{Introduction}

The mechanics of the swimming of microorganisms in the natural world has fascinated researchers over the past 70 years (Purcell 1977; Lauga and Powers 2009) since the work of Taylor (1951). Research interest in this area has expanded rapidly in recent years with the main focus on the design of micro-scale machines (Abbott et al. 2009; ErbasCakmak et al. 2015) for drug delivery in medical applications (Nelson et al. 2010). Two general approaches have emerged in this research. In one, models have been developed which mimic the natural world (Dreyfus et al. 2005; Elgeti et al. 2015; Benkoski et al. 2009, 2011; Cebers and Javaitis 2004) including important effects such as synchronization (Golestanian et al. 2011), boundaries (Lauga et al. 2006) and torque (Darnton et al. 2007). In the other, apparently simple models containing three spheres have been proposed (Najafi and Golestanian 2004; Vladimirov 2013; Felderhof 2006, 2015) with the focus on investigating the basic mechanics of swimming at low Reynolds numbers. A physical model of a three-sphere swimmer has recently been investigated (Grosjean et al. 2016) and it has been shown to be capable of self-propulsion on the surface of a fluid.

Our experimental investigations were concerned with the motion of fully submerged sets of coupled spheres in a Stokes flow. It is known that the inclusion of small amounts of fluid inertia is sufficient to generate motion (Klotsa et al. 2015) in coupled spheres, but our investigation concerns Stokes flows where fluid inertial effects can safely be ignored. The couplings between the spheres were formed by thin struts which were either rigid or elastic. Similar systems have been studied previously using a few spheres (Farzin et al. 2012; Wang and Othmer 2015; Keaveny and Maxey 2008; Taghiloo and Miri 2013) or a large number of 
spheres to mimic the 'tail' of a swimmer (Roper et al. 2006, 2008; Gauger and Stark 2006). Our objective was rather different and addressed the question of how many interacting spheres were required to generate net motion of the centre of mass of the body. This general question is addressed in other investigations of the motion of spheres in Stokes flows where it is shown that three interacting spheres will give rise to chaotic motion and collective movement of the centre of mass of the group (Jánosi et al. 1997; Mullin et al. 2005; Segre et al. 2010).

A simple theoretical model which is capable of selfpropulsion is the three-linked-sphere model (Najafi and Golestanian 2004) which comprises three spheres linked by extendible rods. The middle sphere is an active element which changes the length of the connecting rods. A nonzero phase difference in the continuous, periodic motion of the two rods means that the motion is non-reciprocal and thereby does not violate Purcell's scallop theorem (Purcell 1977; Pak and Lauga 2015), which is a consequence of the time-reversibility of Stokes flows (Taylor 1951; Happel and Brenner 1983). The non-reciprocal motion of the individual spheres results in global motion of the centre of mass and the basic principal has been explored numerically (Earl et al. 2007) in the three-sphere model and its variants (Avron et al. 2005; Earl et al. 2007; Montino and DeSimone 2015). Indeed, a micro-scale realisation of the threesphere system, achieved using optical tweezers to control the motion of glycerol beads, has been used to demonstrate fluid pumping (Leoni et al. 2008) at the micro-scale. This result indicates that the configuration is capable of self-propulsion. However, to realize, such a swimmer in practice requires control of the motion of each of the two connecting rods.

Our goal was to enable non-reciprocal motion of the swimmer using appropriate design of the geometry rather than individual control of the constituent elements. Our three-sphere swimmers comprised three rigid spheres linked by two soft, thin elastic struts. It is well established that an important ingredient of self-propulsion is internal torque generation (Darnton et al. 2007). We created torques using embedded permanent magnets and applied a modulated uniform external field to induce motion. Magnetic fields have been used in the past to drive swimmers (Keim et al. 2012; Gilbert et al. 2011a, b; Ogrin et al. 2008), but they typically use 'soft' magnetic materials which rely on internal field generation. More generally, field gradients have also been used to guide swimming robots (Yesin et al. 2006).

We used hard magnetic materials, so that the action is purely torque generation through alignment of the magnets with the applied field. The magnets do not break the symmetry of the system; instead, the asymmetry arises, because the struts are unequal in length. When the two end spheres were forced to rotate by the magnetic field, they caused the elastic struts to buckle, and interact with each other through a combination of elastic and fluid forces. On application of an oscillatory field, the asymmetry in the length of the 'arms' of the swimmer resulted in non-reciprocal body motion which induced self-propulsion. We conducted our investigation at the macro-scale: the device was centimetres long and hence it was immersed in a very viscous oil to enable close approximation to Stokes flow. We chose to work in this way as it enabled detailed observation of the swimmer and any fluid motion.

We first outline the experimental apparatus and report results for devices comprising two and three linked spheres. We show that whereas the motion of a device containing two spheres is reciprocal and there is no net motion, that for a three-sphere model is non-reciprocal and self-propulsion is found. We conclude that the hydrodynamic and elastic interactions between the spheres in the swimmer are able to generate non-reciprocal body motion which leads to self-propulsion at low Reynolds number, which here we define to be $R e=a u_{\max } / \nu<1$, where $a$ is the radius of the spheres, $u_{\max }$ is the maximum velocity of the flow, and $v$ is the kinematic viscosity of the fluid. This work, therefore, demonstrates the proof of concept that a driven array of three linked spheres can self-propel at low Reynolds numbers.

\section{Experimental setup}

The motion of linked spheres in a very viscous fluid was investigated experimentally. The linked-sphere systems comprised active spheres which contained small neodymium magnets that were acted on by an external oscillatory magnetic field, and passive spheres that contained no magnetic material. Application of a spatially uniform magnetic field created a torque, but no net force, on the embedded magnets in the active spheres, as they rotated to align with the applied field.

A schematic diagram of the experimental system is shown in Fig. 1, and details of the experimental apparatus used for magnetic actuation and flow visualization are given in Box et al. (2015). The connected spheres were in a viscous liquid inside a rectangular tank with internal dimensions of width $125 \mathrm{~mm}$, length $115 \mathrm{~mm}$, and height $200 \mathrm{~mm}$. All spheres used were $2 a=12.70 \mathrm{~mm}$ in diameter and made from polypropylene, with density $946 \mathrm{~kg} \mathrm{~m}^{-3}$. In each configuration, there was at least one active sphere which contained a cylindrical neodymium magnet of length $3 \mathrm{~mm}$ and diameter $2.4 \mathrm{~mm}$, and magnetic moment $m=0.0140 \pm 0.0006 \mathrm{Am}^{2}$, embedded within it. Hence, the magnet occupied $<2 \%$ of the volume of each sphere. Further ballast in the form of $0.5 \mathrm{~mm}$ diameter, stainless 


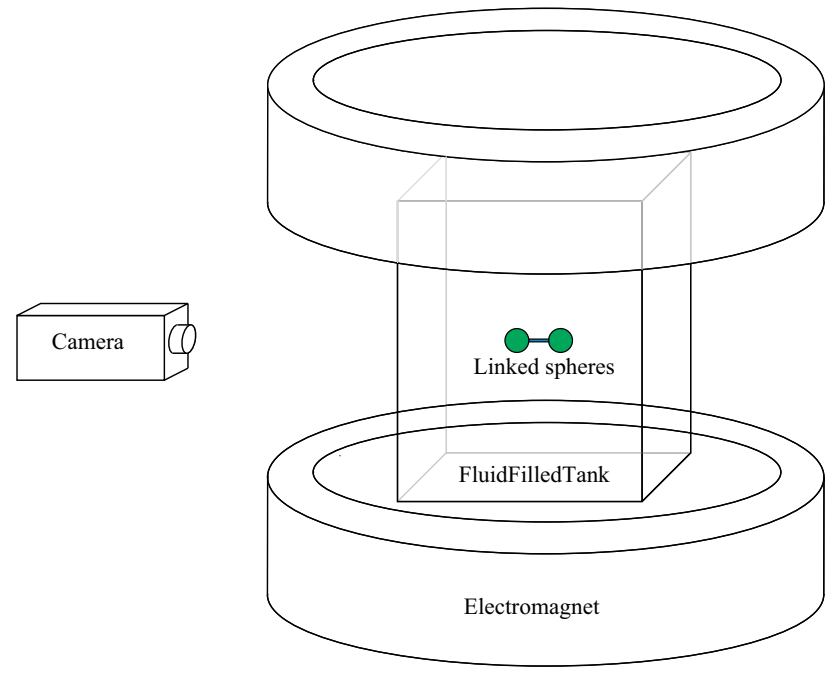

Fig. 1 Schematic diagram of the experimental apparatus. Systems of connected, near neutrally buoyant spheres in a viscous fluid filled tank were subject to a spatially uniform, oscillatory magnetic field applied using Helmholtz coils. The experimental system was contained within a Mumetal canister to reduce the effects of extraneous fields

steel balls were glued to the surface to make the device neutrally buoyant. It was found that these smaller spheres had a negligible effect on the motion. Passive spheres had small strips of copper wire embedded within them, and again, small stainless steel balls were used to achieve neutral buoyancy.

The fluid was silicone oil with a measured kinematic viscosity of $v=922 \pm 1 \mathrm{~mm}^{2} \mathrm{~s}^{-1}$ and density of $\rho=975 \pm 1 \mathrm{~kg} \mathrm{~m}^{2}$ at $20.0{ }^{\circ} \mathrm{C}$. The kinematic viscosity and density of the silicone oil were calibrated as a function of temperature, using a suspended level viscometer and a hydrometer, respectively, so that variations in temperature between experiments could be accounted for. Furthermore, experiments were performed in a air-conditioned laboratory where the room temperature was maintained at $20.0 \pm 1^{\circ}$ $\mathrm{C}$, and the mean recorded temperature inside the tank was found to be $19.89 \pm 0.30{ }^{\circ} \mathrm{C}$.

The applied magnetic field was generated using Helmholtz coils and was measured to be spatially uniform to within $1 \%$. A Mumetal shield surrounded the apparatus to minimize the effects of background magnetic fields. The embedded magnets were initially set with their axes in a direction orthogonal to the applied field. As the external field was increased, the embedded magnets became aligned with it, so that a torque acted on the magnets in the spheres. Experiments performed on pairs of linked spheres were conducted for a field strength of $B=1.5 \mathrm{mT}$ and a frequency of $f=0.5 \mathrm{~Hz}$, while experiments performed on three linked spheres were conducted for $B=1.1 \mathrm{mT}$ and $f=0.5 \mathrm{~Hz}$.
A measure of the ratio of viscous torque to magnetic torque acting on a magnetically actuated sphere in a fluid environment is provided by the dimensionless Mason number $M n=8 \pi \mu a^{3} \omega / B m$, where $\mu$ is the dynamic viscosity of the fluid, $a$ is the radius of the sphere, $\omega=2 \pi f$ and $B$ are the angular frequency and magnitude of the applied magnetic field, respectively, and $m$ is the magnetic moment of the magnets embedded within the sphere. In the case of the two-sphere devices, $M n=0.86$, and in the case of the three-sphere device, $M n=1.18$. In both cases, magnetic forcing and viscous damping provided comparable contributions to the resultant motion of the devices. We may also calculate the Womersley number $\alpha=a(\omega / \nu)^{1 / 2}$ to compare the contributions of transient inertial forces to viscous shear forces. Here, we find that $\alpha=0.37$ which indicates that inertial effects are small compared to viscous effects.

The motion of the linked spheres was measured from a sequence of images captured on a Genie camera (HM1400, Teledyne DALSA, Canada) with a spatial resolution of $0.12 \mathrm{~mm} /$ pixel, at a rate of $50 \mathrm{~Hz}$ and with $640 \times 640$ pixels. In the case of the three-linked spheres, Particle Image Velocimetry (PIV) measurements were also performed to measure the induced flow field. A high-speed camera (pco.1200 hs, PCO AG, Kelheim, Germany) with a spatial resolution of $0.05 \mathrm{~mm} /$ pixel was used to image the region of interest. The camera was positioned orthogonal to the $y-z$ plane. A continuous $50 \mathrm{~mW}$ laser sheet illuminated the plane from above, whilst an Nd:YAG pulsed laser sheet lit the plane from below. The camera was synchronised with the Nd:YAG laser using a pulse generator (BNC Model 500, Oxford Lasers Ltd., Oxon, UK) and imaged with $1280 \times 1024$ pixels at a rate of $15 \mathrm{~Hz}$, the maximum pulse rate of the Nd:YAG laser, and with an exposure of between 10 and $20 \mathrm{~ms}$. A low-pass filter was positioned between the tank and the camera to reduce background noise in the detected signal. A consequence of the maximum pulse rate of the laser was that PIV measurements were performed for a frequency of applied magnetic field of $0.15 \mathrm{~Hz}$ for which $M n=0.35$ and $\alpha=0.20$.

The pairs of linked spheres were connected either by glass or rubber links, as shown in Fig. 2a and b, respectively. The glass rods were of circular cross section, of diameter $1.8 \pm 0.1 \mathrm{~mm}$ and lengths $l=2.8 \pm 0.1,6.9 \pm 0.1$ , and $12.7 \pm 0.1 \mathrm{~mm}$. The elastic struts were made from silicone rubber, a linearly elastic material with a Young's Modulus of $\sim 1 \mathrm{MPa}$ (Singh et al. 2013; Tipton et al. 2012), with a $1.2 \pm 0.1 \mathrm{~mm}$ square cross section, and lengths of $l=3.2 \pm 0.1,6.1 \pm 0.1,10.0 \pm 0.1,15.0 \pm 0.1,21.0 \pm 0.1$, and $25.2 \pm 0.1 \mathrm{~mm}$. The ends of each connector were glued to the surfaces of the spheres, such that the connector was approximately parallel to the axis of magnetic dipole of the active sphere. 

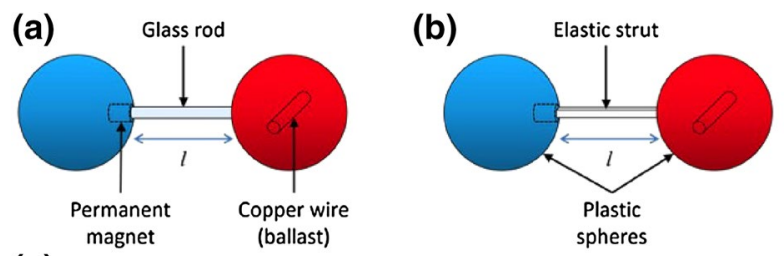

(c)

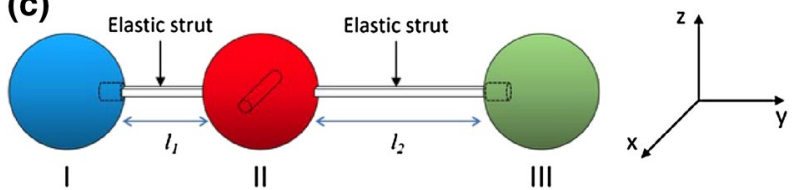

Fig. 2 Schematic diagram of the linked two-sphere systems comprising two spheres linked by $\mathbf{a}$ a glass rod and $\mathbf{b}$ an elastic strut of length $l$. The active spheres contain a neodymium magnet that was actuated by the applied magnetic field, and the non-magnetic passive spheres were carefully weighted with copper to ensure near-neutral buoyancy of the system. c The swimmer comprised of three spheres connected by elastic struts of unequal length $l_{2}>l_{1}$. Permanent magnets were embedded in the active, end spheres (I and III) and the passive, middle sphere (II) was weighted with copper wire. The dipoles of the magnets were aligned in the $y$-direction, and the external magnetic field was in the $z$-direction. The dipoles in sphere I and III were in the opposite directions. Observations were made by viewing the motion in the $x$-direction

The three-linked-sphere device comprised $12.7 \pm 0.01 \mathrm{~mm}$ spheres connected to each other by two thin, silicone rubber struts in the configuration shown in Fig. 2c. In practice, a perfectly symmetrical system is impossible to manufacture. Here, an asymmetry was deliberately introduced into the system using elastic struts of different lengths. Both elastic struts had a $1.2 \pm 0.1 \mathrm{~mm}$ square cross section, one was $3.0 \pm 0.2 \mathrm{~mm}$ long and the other $6.0 \pm 0.2 \mathrm{~mm}$, such that the total length of the swimmer was $l_{b}=6 a+l_{1}+l_{2}=47.1 \mathrm{~mm}$. Both the end spheres contained a single magnet with their dipoles set in opposite directions, such that the torque induced by the external field created clockwise and anti-clockwise rotation in the spheres at either end of the device. The opposing torques bent the connecting struts attached to the passive central sphere.

Since it is known that nearby walls significantly affect the motion of swimmers (Lauga et al. 2006; Berke et al. 2008), we considered the separation distance $h$ between the swimmer and the walls of the tank. For spheres performing torsional oscillations near stationary boundaries, the viscous resistance introduced by the presence of a wall is negligible provided the separation distance is greater than the thickness of the viscous boundary layer on the wall, independent of the orientation of the rotational axis of the sphere with respect to the surface normal (Box et al. 2017). The thickness of the Stokes boundary layer $\delta=(v / \omega)^{1 / 2}$, which gives a measure of the distance over which the amplitude of fluid motion decays to $1 / e$ of the initial value, was estimated to be $2.69 a$ for typical measurements and $4.93 a$ for the PIV measurements. In both cases, this was less than the minimum distance separating the swimmer from the walls of the tank $\delta<h_{\min }=6.13 a$, such that the confining boundaries can be considered to be sufficiently far away to have had negligible effect on the device. Experiments on the swimming device were also performed in a circular tank of diameter $300 \mathrm{~mm}$ and height $200 \mathrm{~mm}$, and the behaviour of the device was found to be quantitatively comparable in both cases.

\section{Two linked spheres}

Results are first presented from an investigation into the motion of pairs of spheres connected by thin connectors. In each case, one sphere was made active by embedding a neodymium magnet and driving it using an external oscillatory field, whilst the other was passive. Results from pairs of linked spheres are compared and contrasted where the connectors have different lengths and they were either rigid or flexible.

\subsection{Glass connector}

The simplest orbits were exhibited by the spheres connected by rigid glass rods. The induced magnetic torque of the active sphere caused the system to pivot about its collective centre of mass which was located approximately half-way along the connecting rod. Hence, the two spheres performed a see-saw motion about this fulcrum, each sphere prescribing an arc-like orbit through the fluid. The qualitative shape of the trajectories of the spheres was found to be independent of the length of the connector connecting the spheres. The trajectories shown in Fig. 3 are the positions, in $y-z$ coordinates, of the respective centres of mass of the active and the passive sphere through 8 periods of oscillation when connected by a glass rod of length $l=0.44 a$.

\subsection{Elastic connector}

The flexibility of the elastic connector resulted in the active and passive spheres prescribing trajectories that were qualitatively different for short and long connectors. For short connectors, the orbits are similar to the rigid connectors, as shown in Fig. 4, for an elastic strut of length $l=0.50 a$. For longer elastic connectors, the orbits become more complex, as can be seen in Fig. 5. The increased flexibility of the strut permitted more bending. Hence, the applied torque bent the connector rather than displacing the spheres. As a result, the passive sphere had a smaller orbit and travelled a 

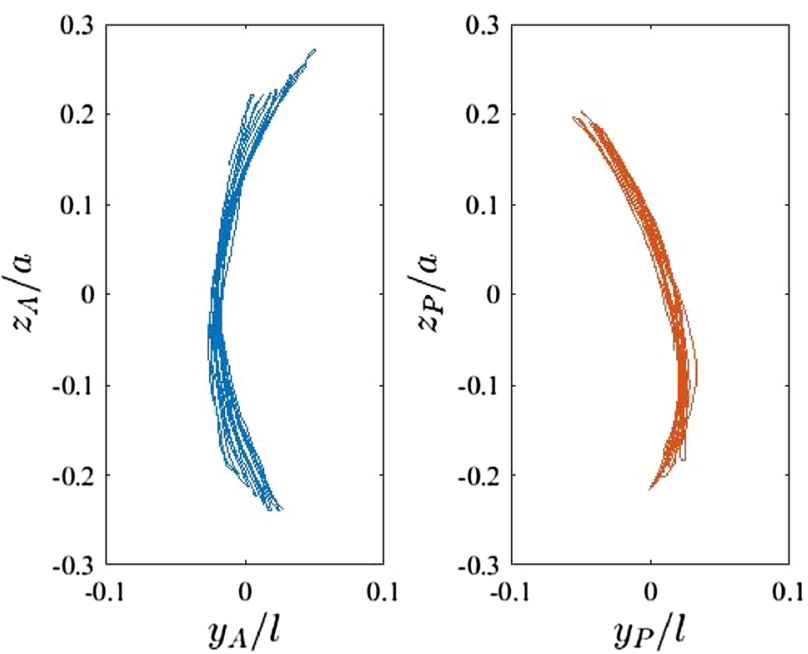

Fig. 3 Orbits of the active (left) and passive (right) spheres, connected by a rigid glass rod of length $l=0.44 a$, throughout 8 periods of oscillation
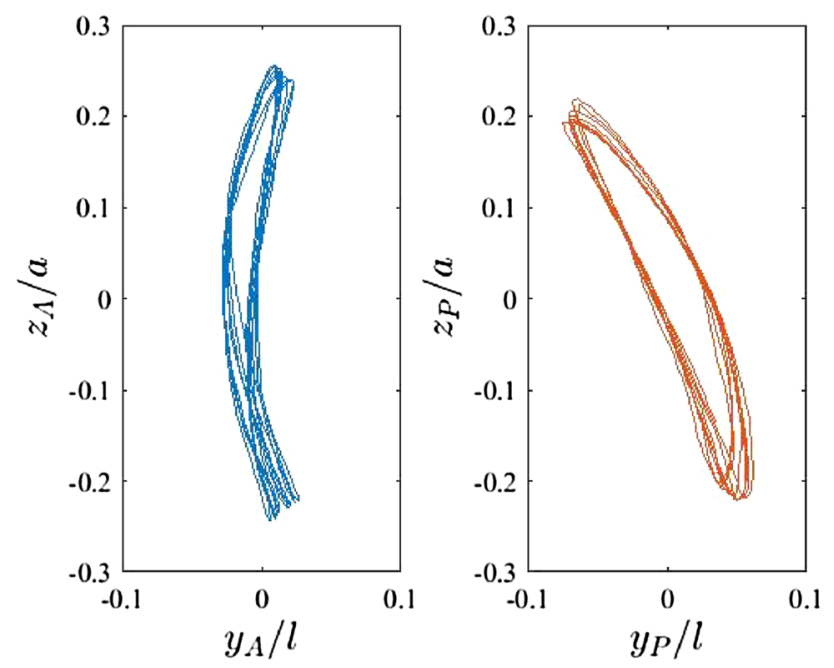

Fig. 4 Orbits of the active (left) and passive (right) spheres, connected by an elastic strut of length $l=0.50 a$, throughout 8 periods of oscillation

shorter distance in one cycle as the length of the connector increased.

The bending of the connector introduced a phase delay between the motion of the spheres, such that the orbit of the passive sphere lagged the driven sphere. However, the bending resistance introduced a restoring force which maintained the separation between the spheres.

\subsection{Comparison of rigid and non-rigid links}

The distance travelled in one orbit by the individual active and passive sphere, $D / a$, measured as a function of
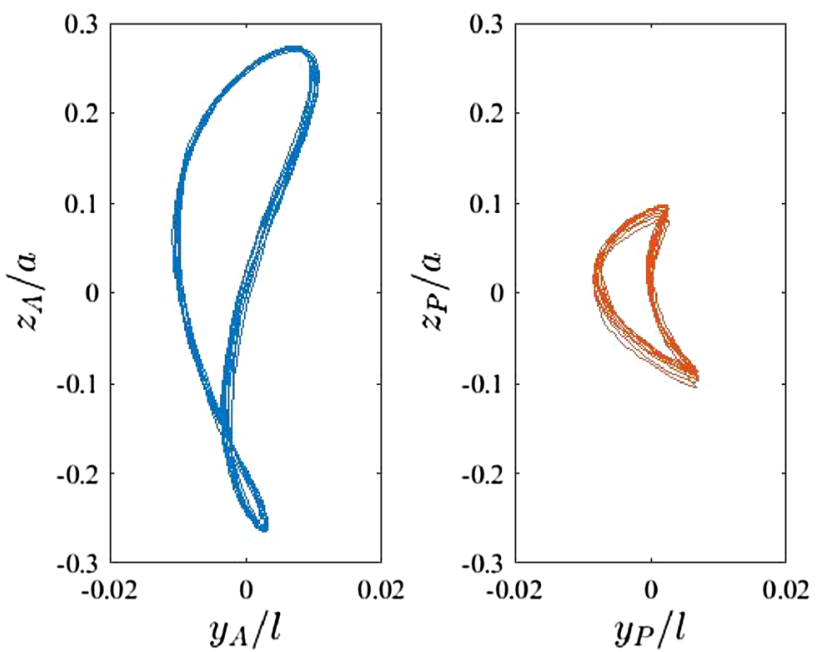

Fig. 5 Orbits of the active (left) and passive (right) spheres, connected by an elastic strut of length $l=3.97 a$, throughout 10 periods of oscillation

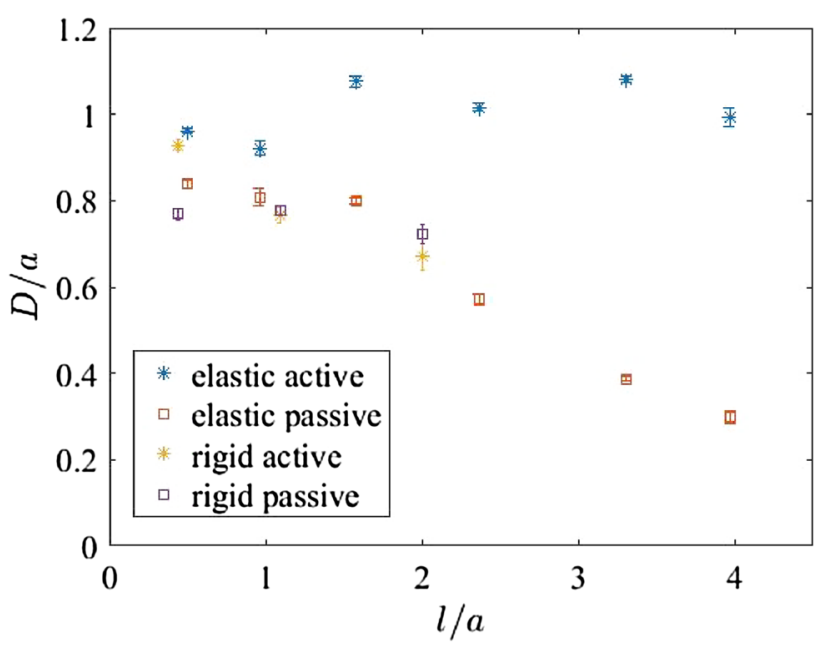

Fig. 6 Distance travelled by the individual active and passive sphere in one orbit, $D / a$, measured as a function of length of the connection $l / a$, between the two spheres for both rigid and flexible links (as indicated in the legend)

connector length, $l / a$, is shown in Fig. 6 for both elastic and rigid connections. In both cases, the total distance travelled in one cycle by both the active and the passive sphere increased with decreasing length of connecting connector, which suggests that larger connectors are subjected to greater viscous resistance. The increased flexibility of the long elastic connections permitted increased bending of the connector which resulted in the passive sphere prescribing a smaller orbit. 


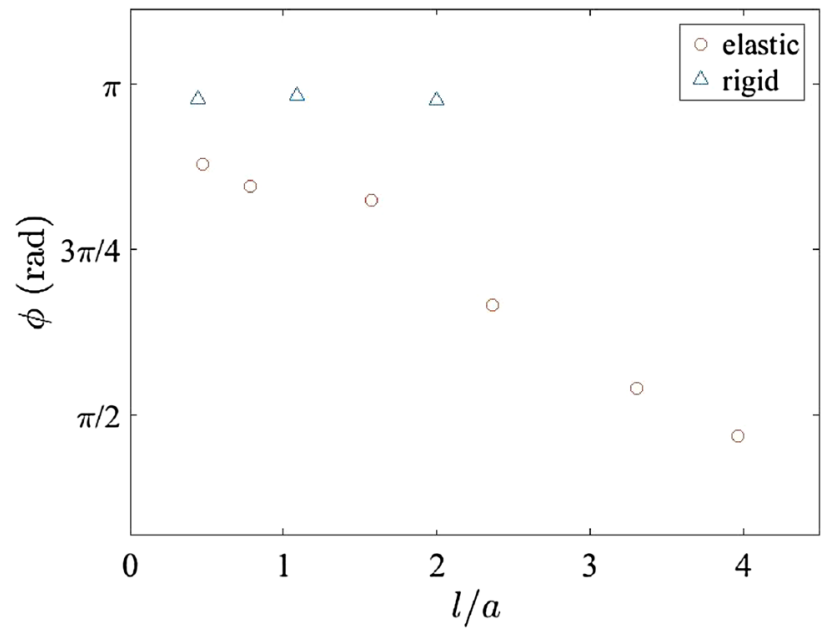

Fig. 7 Phase delay, $\phi$, between the torsional oscillation of the active sphere and the displacement of the passive sphere as a function of the length of connection, $l / a$, for elastic connectors and rigid glass connectors (as indicated in the legend)

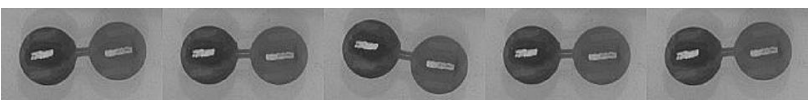

Fig. 8 Five stages of the reciprocal oscillation cycle performed by two spheres connected by a rigid, glass rod of length $l=0.28 a$. The time interval between each stage of the oscillation cycle is $\tau=0.79$

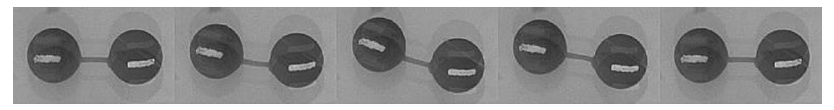

Fig. 9 Five stages of the reciprocal oscillation cycle performed by two spheres connected by a flexible, elastic strut of length $l=0.96 a$. The time interval between each stage of the oscillation cycle is $\tau=0.79$

The displacement of the passive sphere connected by rigid glass is found to be approximately in antiphase with the torsional oscillation of the active sphere, ${ }^{1}$ independent of length of the connection. This implies that the active and passive spheres were in phase throughout a cycle. However, the flexibility of the elastic connections caused the response of the passive sphere to lag the torsional motion of the active sphere. Furthermore, the phase difference increased with increasing length of flexible connector, as shown in Fig. 7.

\footnotetext{
${ }^{1}$ We have used the convention that a positive torsional oscillation is in the clockwise direction and positive displacement of the passive sphere is in the positive $z$-direction.
}

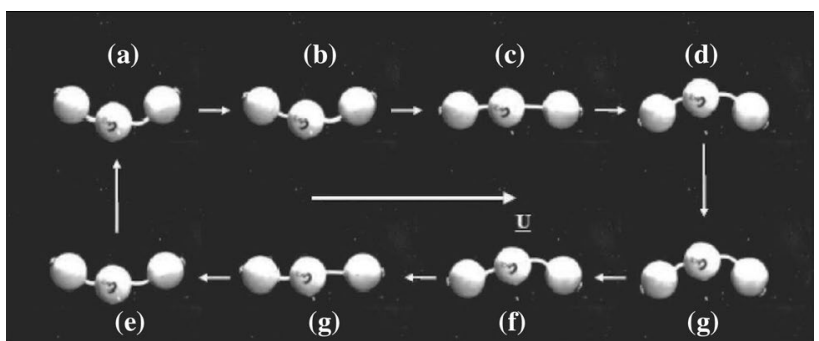

Fig. 10 Eight stages of the non-reciprocal buckling cycle of the swimmer are shown below. The large, white arrow in the centre of the images denotes the direction of the velocity of the swimmer

The motion of two connected spheres was reciprocal, independent of the type of link, as can be seen for rigid links in Fig. 8 and for flexible links in Fig. 9. This implies that the motion of two linked spheres would not result in self-propulsion at low Re Purcell (1977). Although it must be noted that if two spheres were connected by a sufficiently long and flexible connector then, on actuation of the active sphere, bending waves would propagate along the connector towards the passive sphere (Lauga 2007; Wiggins et al. 1998; Yu et al. 2006). The non-reciprocal motion of such a connector, and the flow it could induce, may result in the propulsion of the configuration.

\section{Swimmer}

In this section, the understanding gained from studying the interaction of connected active and passive sphere pairs is used to provide insight into the locomotive mechanism of a magnetically actuated swimmer. The swimmer comprised an arrangement of active and passive spheres connected with elastic struts of unequal length and was observed to self-propel in a Stokes flow.

The magnetic-dipole axes of the two active, end spheres were aligned orthogonal to the applied field, and the dipole moments of the two magnets were set opposite, such that the torque induced by the applied field resulted in the rotation of the end spheres in opposite directions. Rotation of the end spheres caused the middle sphere to be displaced vertically. However, the end spheres did not just perform rotary oscillations about a fixed point in the fluid. As a consequence of hydrodynamic interactions between the three spheres and the restoring force of the connecting struts, the two end spheres prescribed trajectories in the fluid whilst simultaneously performing torsional oscillations. Furthermore, the inequality in length of elastic struts introduced a small phase difference between the response of the middle and end spheres.

The net effect of the motion of the two active end spheres and the displaced middle sphere was a buckling of 


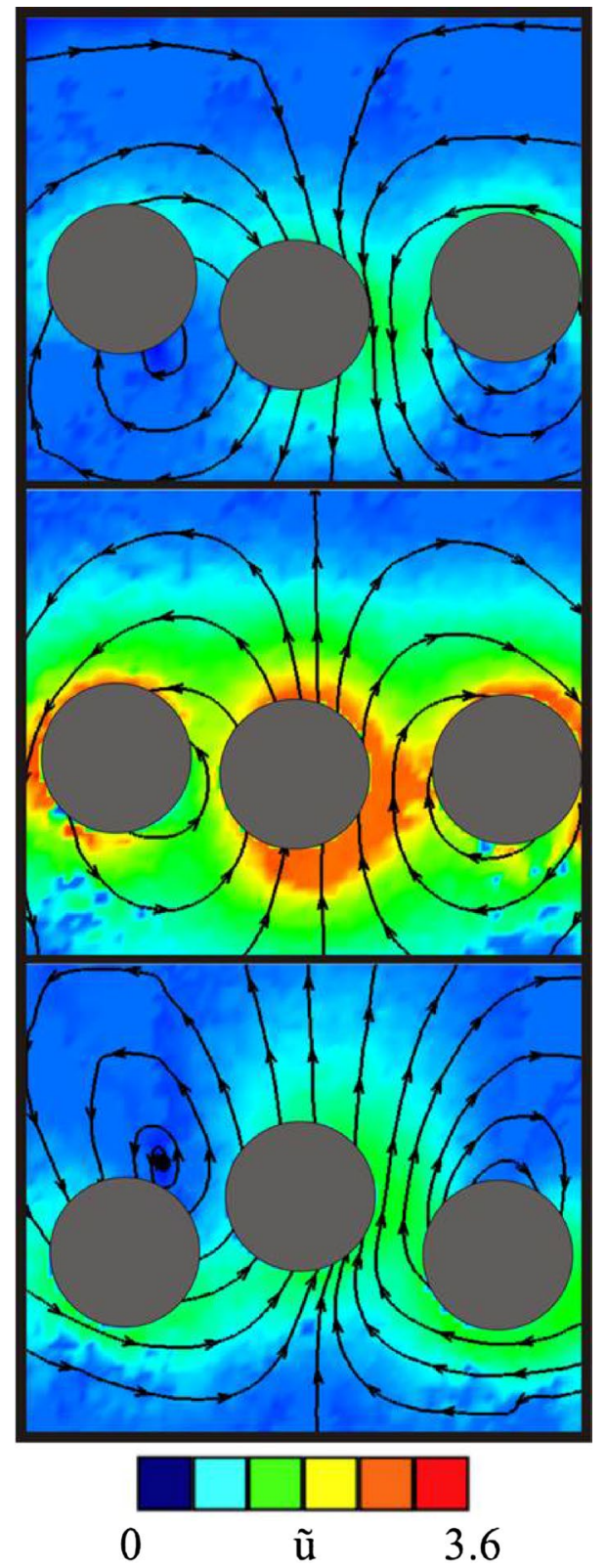

Fig. 11 Flow visualization of the swimmer at $\pi / 2$ (top), $\pi$ (middle), and $3 \pi / 2$ (bottom) in the buckling cycle. The spheres are depicted by the grey circles and the longer strut connects the middle sphere to the right-hand sphere. The black arrowed lines represent estimates of the flowlines and the colour contours represent the magnitude of the velocity, $\tilde{u}=u / a \omega$, which ranges from 0 to 3.6

the swimmer. The swimmer buckled periodically in a nonreciprocal, spatially asymmetric manner which propelled the swimmer in the direction of the longest strut. The buckling cycle is depicted in the sequence of images in Fig. 10. The white arrow in the centre of the image denotes the direction of travel.

The flow fields at three points, separated by $\pi / 2$, in the non-reciprocal buckling cycle are shown in Fig. 11. The
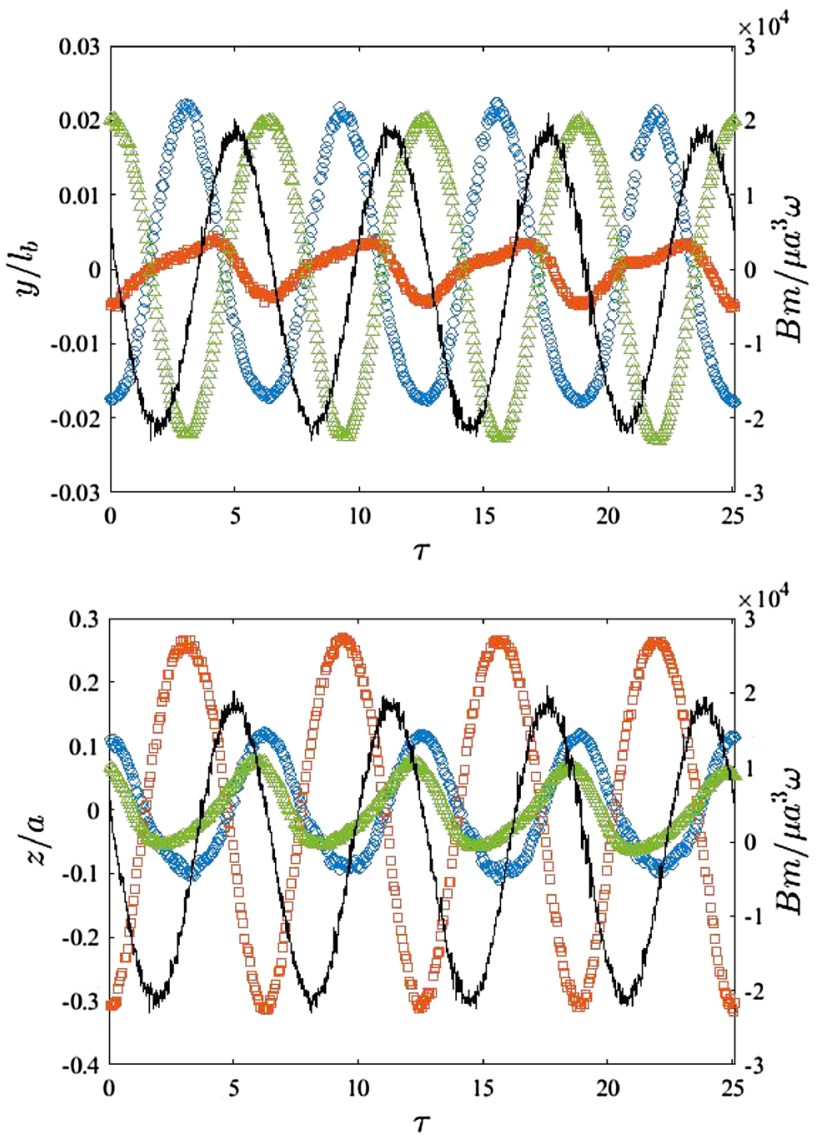

Fig. 12 Time-series of the horizontal displacement (top) and vertical displacement (bottom) of the three spheres. The motion of active sphere I is denoted by the blue circles, passive sphere II by the red squares, and active sphere III by the green triangles. Motion in the horizontal direction is non-dimensionalised by the length of the swimmer, $l_{b}$, and vertical motion is non-dimensionalised by the radius of the spheres which comprise the swimmer, $a$. The non-dimensional applied magnetic field strength $B m / \mu a^{3} \omega$ is represented by the black line

top and bottom images correspond to the maximum buckling of the swimmer body, and the middle image shows the swimmer in an intermediate state. The individual spheres are depicted by the grey circles and the longer strut connects the middle sphere to the right-hand sphere, as in the schematic shown in Fig. 2. The black arrowed lines indicate instantaneous flowlines and the magnitude of the fluid velocity is represented by the colour contours. The spatial asymmetry in the flow field is evident throughout the buckling cycle of the swimmer. In the top and bottom images, in particular, more flowlines are directed towards the sphere connected by the longer strut (sphere III). Furthermore, the magnitude of the fluid velocity is consistently greater adjacent to the longer strut throughout the cycle.

A set of time-series of the applied magnetic field and the motion of each sphere in the horizontal and vertical direction are shown in Fig. 12. Following the convention 

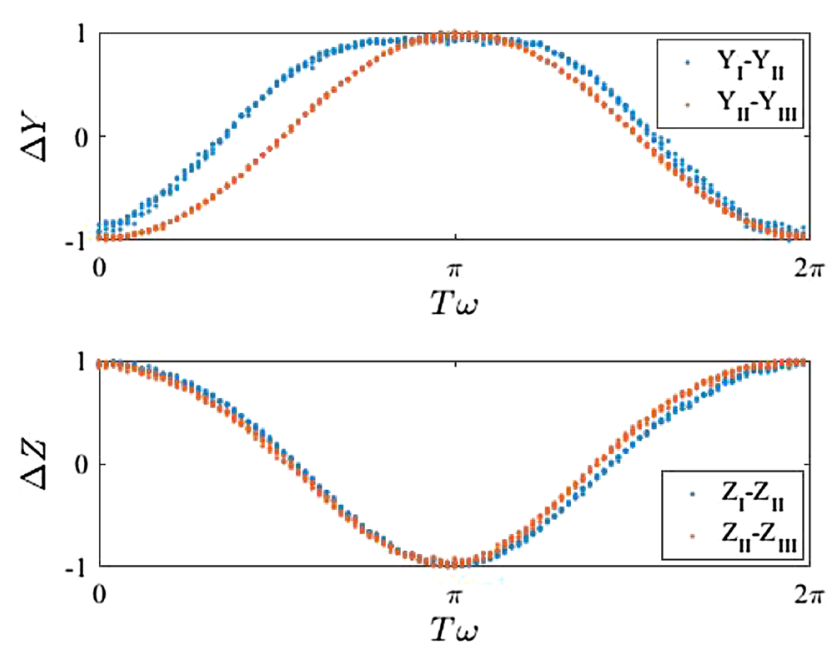

Fig. 13 Normalised distance between spheres over one period, $T=2 \pi / \omega$, of the buckling cycle of the swimmer. (Top) Horizontal distance between spheres I and II, $\Delta Y=Y_{\mathrm{I}}-Y_{\mathrm{II}}$, and spheres II and III, $\Delta Y=Y_{\text {II }}-Y_{\text {III }}$. (Bottom) Vertical distance between spheres I and II, $\Delta Z=Z_{\mathrm{I}}-Z_{\mathrm{II}}$, and spheres II and III, $\Delta Z=Z_{\mathrm{II}}-Z_{\mathrm{III}}$. The separation distance is normalised by the peak values, such that the maximum and minimum separations are given by 1 and -1 , respectively, and $T=0 \mathrm{~s}$ is set, such that it corresponds to the maximum buckling of the swimmer. The data shown here are the same as those shown in Fig. 12

depicted in Fig. 2, the data for the spheres are denoted by the blue, red, and green points, respectively. The horizontal motion of the two active, end spheres are in antiphase (and delayed with respect to the applied field by $\sim \pi / 4$ ) which suggests a longitudinal compression and relaxation of the swimmer about its centre.

A better understanding of the motion of the spheres can be obtained by examining their separation, as shown in Fig. 13. Here, we plot the horizontal separation $\Delta Y$ and the vertical separation $\Delta Z$ between the sphere I and sphere II, and between sphere II and sphere III, over the period $T$ of a buckling cycle. The separation distance is normalised by the peak values, such that the maximum and minimum separations are given by 1 and -1 , respectively, and the beginning of the period of the buckling cycle is set, such that it corresponds to maximum buckling of the swimmer.

It can be inferred from these results that in the first half of the period, the swimmer straightens and the horizontal separation between spheres I and II reaches its maximum value before that of spheres II and III by approximately $T \omega=0.8 \pi$. Whereas, in the second half of the period, the swimmer buckles in the opposite direction and the reduction in horizontal separation between spheres I and II lags that of spheres II and III, although the lag is much smaller than in the first half of the period. Over the period of the buckling cycle, a compressive wave travels along

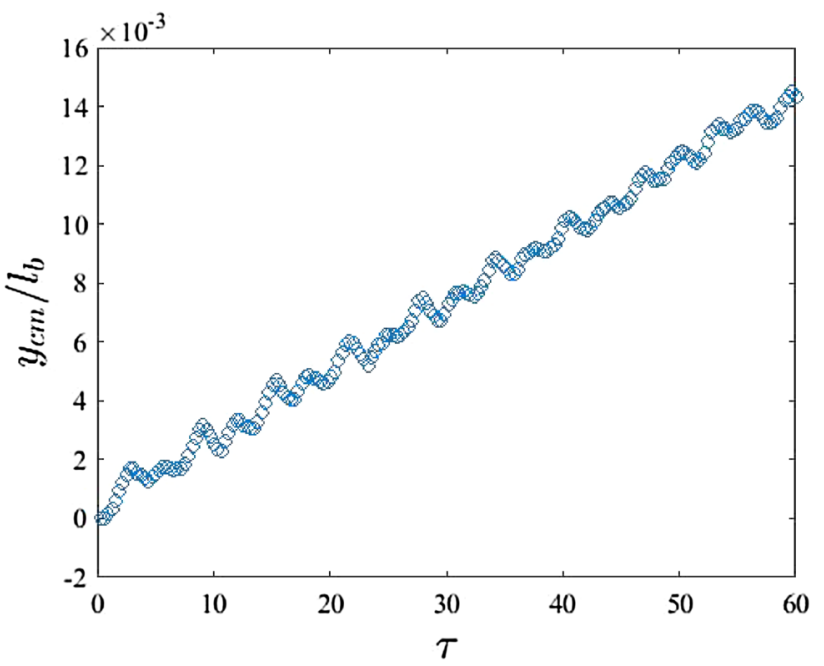

Fig. 14 Centre of mass of the swimmer $y_{\mathrm{cm}} / l_{b}$, non-dimensionalised by the length of the swimmer $l_{b}$, measured as a function of time $\tau=t \omega$

the length of the swimmer in the direction of the longer strut and the longitudinal motion of the multi-body configuration is non-reciprocal. It can also be inferred that there is no significant phase difference in the vertical direction, although there is an asymmetry in the vertical motion of the end spheres. The vertical motion of the middle sphere is, however, approximately in antiphase to the other two spheres which confirms that the rotation of the end spheres drags the middle sphere through the fluid.

The resultant horizontal motion of the centre of mass of the swimmer is shown as a function of time in Fig. 14. The swimmer was observed to move with a non-dimensional speed of $\tilde{v}=v / l_{b} \omega=2.03 \times 10^{-4}$ at $\operatorname{Re} \sim 0.1$ and for $M n=1.18$. The swimmer was also observed to selfpropel at $R e \sim 10^{-4}$ in a more viscous fluid of viscosity $v \sim 1.4 \times 10^{4} \mathrm{~mm}^{2} \mathrm{~s}^{-1}$. In this case, the propelling rate was $\tilde{v}_{\text {visc }}=9.01 \times 10^{-6}$ and $M n_{\text {visc }}=0.09$. We find that $2\left(M n / M n_{\text {visc }}\right) \sim \tilde{v} / \tilde{v}_{\text {visc }}$ which indicates that the rate of self-propulsion was governed by a balance between the induced magnetic torque and the viscous resistance to motion acting on the two end spheres, and that the locomotive mechanism was maintained when the configuration was a closer approximation to a Stokes flow.

Finally, we note that attempts at fabricating a symmetrical swimmer, comprising three spheres connected by elastic struts of equal length, were made. Small asymmetries in the configuration remained, as is inevitable in any physical system, and resulted in swimming but at a significantly reduced velocity. 


\section{Summary and discussion}

The results of an investigation into the effects of linking driven and passive spheres have been presented. Pairs of spheres connected by rigid links oscillated in phase throughout a cycle. However, the introduction of flexible connectors introduced a phase delay between the actuation of the active sphere and the response of the passive sphere. The phase delay increased with increasing length of the connector. Longer connectors were also subject to more viscous resistance, and longer elastic connectors also bent more.

A swimmer, comprising three spheres connected by elastic struts of unequal length, was observed to deform in a non-reciprocal manner and propel itself through the viscous fluid in the direction of the longest strut. The spatial asymmetry gives rise to non-reciprocality in the buckling cycle of the swimmer. The inequality of strut length introduced a phase difference between the actuation of the two arms of the swimmer. Specifically, the middle sphere is influenced by the rotation of the sphere connected by the shorter strut first. As in the Purcell threelink swimmer (Purcell 1977) and the Najafi-Golestanian three-sphere swimmer (Najafi and Golestanian 2004; Leoni et al. 2008), the phase difference between the motion of the arms of the swimmer meant that the periodic sequence of shapes displayed by the swimmer was not kinematically reversible.

A common feature of propulsion mechanisms at low$R e$ is the propagation of a deforming wave along the body of a swimmer. Swimmers which move in the direction of wave propagation are referred to as pullers, whereas pushers move in a direction opposite to the direction of wave propagation (Pak and Lauga 2015). We found that a compressive wave propagated along the swimmer body from sphere I to sphere III, over the period of one buckling cycle. The compressive wave propagated in the same direction as the self-propulsion of the swimmer. In an attempt to better understand the propulsion mechanism, we compare the swimmer to the far-field description of a puller, which is a negative Stokes dipole. This suggests that the swimmer drags itself through the fluid by drawing in fluid from the ends, along the swimming direction, and ejecting fluid from the sides.

This novel approach to swimmer design enables the development of a range of multi-body configurations of oscillating spheres. Furthermore, as the actuation of the swimmer is controlled completely by the interaction of hard magnets and an external field, it will be possible to reproduce the swimmer at the micro-scale. Such a synthetic swimmer has potential for implementation in in vivo drug delivery (Gao et al. 2012) and as pumps in microfluidic devices (Leoni et al. 2008).
Acknowledgements The authors are grateful to Prof. Sir Konstantin Novosolev FRS for the loan of the electro-magnet.

Open Access This article is distributed under the terms of the Creative Commons Attribution 4.0 International License (http:// creativecommons.org/licenses/by/4.0/), which permits unrestricted use, distribution, and reproduction in any medium, provided you give appropriate credit to the original author(s) and the source, provide a link to the Creative Commons license, and indicate if changes were made.

\section{References}

Abbott JJ, Peyer KE, Lagomarsino MC, Zhang L, Dong L, Kaliakatsos IK, Nelson BJ (2009) How should microrobots swim? Int J Robot Res 28:1434

Avron JE, Kenneth O, Oaknin DH (2005) Pushmepullyou: an efficient micro-swimmer. New J Phys 7:234

Benkoski JJ, Deacon RM, Land HB, Baird LM, Breidenich JL, Srinivasan R, Clatterbaugh GV, Kengb PY, Pyun J (2009) Dipolar assembly of ferromagnetic nanoparticles into magnetically driven artificial cilia. Soft Matter 6:602-609

Benkoski JJ, Breidenich JL, Uy M, Hayes AT, Deacon RM, Land HB, Spicer JM, Kengb PY, Pyun J (2011) Dipolar organization and magnetic actuation of flagella-like nanoparticle assemblies. J Mater Chem 21:7314

Berke AP, Truner L, Berg HC, Lauga L (2008) Hydrodynamic attraction of swimming microorganisms by surfaces. Phys Rev Lett 101:038102

Box F, Singh K, Mullin T (2017) The interaction between oscillating spheres and solid boundaries in a Stokes flow (in preparation)

Box F, Thompson AB, Mullin T (2015) Torsional oscillations of a sphere in a Stokes flow. Exp Fluids 56:209

Cebers A, Javaitis I (2004) Bending of flexible magnetic rods. Phys Rev E 69:021404

Darnton NC, Turner L, Rojevsky S, Berg HC (2007) On torque and tumbling in swimming Escherichia coli. J Bacteriol 189:1756-1764

Dreyfus R, Baudry J, Roper ML, Fermigier M, Stone HA, Bibette J (2005) Microscopic artificial swimmers. Nature 437:862-5

Earl D, Pooley CM, Ryder JF, Bredberg I, Yeomans JM (2007) Modeling microscopic swimmers at low Reynolds number. J Chem Phys 126:064703

Elgeti J, Winkler RG, Gompper G (2015) Physics of microswimmerssingle particle motion and collective behavior: a review. Rep Prog Phys 78:056601

Erbas-Cakmak S, Leigh DA, McTernan CT, Nussbaumer AL (2015) Artificial molecular machines. Chem Rev 115:10081-10206

Farzin M, Ronasi K, Najafi A (2012) General aspects of hydrodynamic interactions between three-sphere low-Reynolds-number swimmers. Phys Rev E 85:061914

Felderhof BU (2006) The swimming of animalcules. Phys Fluids 18:063101

Felderhof BU (2015) Efficient swimming of an assembly of rigid spheres at low Reynolds number. Eur Phys J E 38:90

Gao W, Kagan D, Pak OS, Clawson C, Campuzano S, ChuluunErdene E, Shipton E, Fullerton EE, Zhang L, Lauga E, Wang J (2012) Cargo-towing fuel-free magnetic nanoswimmers for targeted drug delivery. Small 8(3):460-467

Gauger E, Stark H (2006) Numerical study of a microscopic artificial swimmer. Phys Rev E 74:021907

Gilbert AD, Ogrin FY, Petrov PG, Winlove CP (2011) Theory of ferromagnetic microswimmers. Q J Appl Math 64:239-263 
Gilbert AD, Ogrin FY, Petrov PG, Winlove CP (2011) Motion and mixing for multiple ferromagnetic microswimmers. Eur Phys $\mathbf{J}$ E 34:121

Golestanian R, Yeomans JM, Uchida N (2011) Hydrodynamic synchronization at low Reynolds number. Soft Matter 7:3074

Grosjean G, Hubert M, Lagubeau G, Vandewalle N (2016) Realization of the Najafi-Golestanian microswimmer. arXiv:p.1606.08680v1

Happel J, Brenner H (1983) Low Reynolds number hydrodynamics. Martinus Nijhoff, The Hague

Jánosi IM, Tél T, Wolf DE, Gallas JAC (1997) Chaotic particle dynamics in viscous flows: the three-particle Stokeslet problem. Phys Rev E 56:2858-2868

Keaveny EE, Maxey MR (2008) Spiral swimming of an artificial micro-swimmer. J Fluid Mech 598:293-319

Keim NC, Garcia M, Arratia PA (2012) Fluid elasticity can enable propulsion at low Reynolds number. Phys Fluids 24:081703

Klotsa D, Baldwin KA, Hill RJA, Bowley RM, Swift MR (2015) Propulsion of a two-sphere swimmer. Phys Rev Lett 115:248102

Lauga E, DiLuzio WR, Whitesides GM, Stone HA (2006) Swimming in circles: motion of bacteria near solid boundaries. Biophys $\mathbf{J}$ 90:400

Lauga E (2007) Floppy swimming: viscous locomotion of actuated elastica. Phys Rev E 75:041916

Lauga E, Powers TR (2009) The hydrodynamics of swimming microorganisms. Rep Prog Phys 72:096601

Leoni M, Kotar J, Rosetti A, Cicuta P, Lagomarsino MC (2008) A basic swimmer at low Reynolds number. Soft Matter 5:472-476

Montino A, DeSimone A (2015) Three-sphere low-Reynolds-number swimmer with a passive elastic arm. Eur Phys J E 38:40

Mullin T, Li Y, del Pino C, Ashmore J (2005) An experimental study of fixed points and chaos in the motion of spheres in a Stokes flow. IMA J Appl Math 10:851-862

Najafi A, Golestanian R (2004) Simple swimmer at low Reynolds number: three linked spheres. Phys Rev E 69:062901

Nelson BJ, Kaliakatsos IK, Abbott JJ (2010) Microrobots for minimally invasive medicine. Annu Rev Biomed Eng 12:55-85

Ogrin FY, Petrov PG, Winlove CP (2008) Ferromagnetic microswimmers. Phys Rev Lett 100:218102
Pak OS, Lauga E (2015) Theoretical models of low-Reynolds-number locomotion. In: Duprat C, Stone HA (eds) Fluid-structure interactions in low-Reynolds-number flows, chap. 4. RSC Soft Matter Series. Royal Society of Chemistry, Cambridge, pp 100-167

Purcell EM (1977) Life at low Reynolds number. Am J Phys 45:3-11

Roper ML, Dreyfus R, Baudry J, Fermigier M, Bibette J, Stone HA (2006) On the dynamics of magnetically driven elastic filaments. J Fluid Mech 554:167-190

Roper ML, Dreyfus R, Baudry J, Fermigier M, Bibette J, Stone HA (2008) Do magnetic micro-swimmers move like eukaryotic cells? Proc R Soc A 464:877-901

Segre PN, Weeks ER, Davidheiser JE, Syers P (2010) Complex dynamics of three interacting spheres in a rotating drum. Phys Fluids 22:033305

Singh K, Tipton CR, Han E, Mullin T (2013) Magneto-elastic buckling of an Euler beam. Proc R Soc A 469:20130111

Taghiloo M, Miri M (2013) Three-sphere magnetic swimmer in a shear flow. Phys Rev E 88:023008

Taylor GI (1951) Analysis of the swimming of microscopic organisms. Proc R Soc A 209:447-461

Tipton CR, Han E, Mullin T (2012) Magneto-elastic buckling of a soft cellular solid. Soft Matter 8:6880

Vladimirov VA (2013) On the self-propulsion of an $N$-sphere microrobot. J Fluid Mech 716:R1-R11

Wang Q, Othmer HG (2015) Computational analysis of amoeboid swimming at low Reynolds number. Math Biol Eng 12:1303-1320

Wiggins CH, Riveline D, Ott A, Goldstein RE (1998) Trapping and wiggling: elastohydrodynamics of driven microfilaments. Biophys J 74(2):1043-1060

Yesin KB, Vollmers K, Nelson BJ (2006) Modeling and control of untethered biomicrorobots in a fluidic environment using electromagnetic fields. Int J Robot Res 25:527

Yu TS, Lauga E, Hosoi AE (2006) Experimental investigations of elastic tail propulsion at low Reynolds number. Phys Fluids 18:091701 